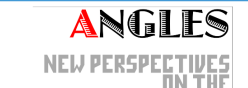

ANELOPHONE WORLD

\section{Angles}

New Perspectives on the Anglophone World

$12 \mid 2021$

COVID-19 and the Plague Year

\title{
Hashtags in Linguistic Anthropology: A COVID-19 Case Study
}

V Shri Vaishali and S. Rukmini

\section{Q OpenEdition}

1 Journals

\section{Electronic version}

URL: https://journals.openedition.org/angles/4489

DOI: 10.4000/angles.4489

ISSN: 2274-2042

\section{Publisher}

Société des Anglicistes de l'Enseignement Supérieur

\section{Electronic reference}

V Shri Vaishali and S. Rukmini, "Hashtags in Linguistic Anthropology: A COVID-19 Case Study", Angles [Online], 12 | 2021, Online since 15 December 2021, connection on 22 December 2021. URL: http:// journals.openedition.org/angles/4489 ; DOI: https://doi.org/10.4000/angles.4489

This text was automatically generated on 22 December 2021.

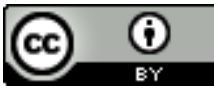

Angles est mise à disposition selon les termes de la Licence Creative Commons Attribution 4.0 International. 


\title{
Hashtags in Linguistic Anthropology: A COVID-19 Case Study
}

\author{
V Shri Vaishali and S. Rukmini
}

\section{Introduction}

1 The COVID-19 outbreak and the eventual lockdowns have brought about everincreasing active social media lives. This can be due to the lack of physical communication and the internet being the only space for communication, information and entertainment. Ruzy Suliza Hazim (2020) has mentioned that "lockdown and selfisolation has led to many people feeling trapped without family and friends. Being cut off from human contact can be mentally draining."(Tan 2020: 13). Digital adoption of everyday life is the coping mechanism that people have identified to avoid the feeling of being trapped. Anita Whiting and David Williams (2013) demonstrated the importance of users and gratification theory concerning social media, and they found ten uses and gratifications: social media interaction, information seeking, pastime, entertainment, relaxation, communicatory utility, convenience utility, expression of opinion, information sharing, and surveillance or knowledge about others. The above ten uses or gratifications play an essential role in coping with COVID-19 lockdowns across borders. From being one of the sources of such gratifications, the Internet in COVID-19 days has become the primary source for the above uses. With the complete dependency on the internet, social media, and digital gadgets for communication, an overwhelmingly pandemic-related content is delivered across social media. So are the hashtagged responses to the pandemic in social media.

2 Hashtags are usually a word or phrase preceded by the symbol '\#' used in social media and digital platforms to find the content under a particular topic. Consequently, hashtags organize the content for the users' needs and help them get every possible content regarding a particular theme or topic in social media. 
Interestingly, the hashtags' organization and categorization roles help create a community of content and services for communicative functions. The recently famous \#blackandwhitechallenge on Instagram is an instance of such communication and community-building functions of hashtags. Michelle Zappavigna (2015) has found that hashtags enact three communicative functions: marking experiential topics, enacting interpersonal relationships, and organizing text. Eventually, the hashtags evolved from being merely used for purposes like business marketing, categorizing and painting a theme on content to become an expression by itself. Hashtags as an expressing device help its users identify the tags as letters of various emotional mind spaces. The user's choice of using hashtags in places like WhatsApp stories where hashtags cannot be tapped to see the other relevant stories is evidence of the identification of hashtags as an emotional expression.

4 This widespread use of hashtags has escalated during the pandemic. As a result, hashtags provided an exciting outlook for academics in understanding the pandemic from the perspective of the humanities and social sciences. The common hashtags used in Facebook and other social platforms, the content style in social media and trending phrases and words related to the pandemic have been recorded, archived and analysed in-depth (Zyot \& Gazo 2021; Nikjoo et al. 2020; Cahapay 2020). Al Azzawi et al. (2021) have studied how people 'speak corona' using hashtags in social media. The presence of bots and other related conspiracies and misinformation using hashtags during COVID-19 has also been studied in detail by numerous researchers (Hussain 2020; Shanthakumar et al., 2020; Chen 2020). While multitudinous research inspects the pandemic life recorded in social media from numerous perspectives, the position of hashtags in understanding people's linguistic behaviour during the pandemic is still underexplored. The anthropological significance of the recent episode of COVID-19 is unquestionable. The present research attempts to study the linguistic patterns of hashtags from an anthropological perspective to understand people's mind space in social media during the pandemic.

\section{Hashtags in Linguistic Anthropology}

5 Linguistic anthropology is the study of human behaviour and culture from a linguistic point of view. Duranti (1997) suggests that linguistic anthropology approaches language through anthropological concerns. Further, he also clarifies the various anthropological concerns linguistics can attend to: "the transmission and reproduction of culture, the relationship between cultural systems and different forms of social organisation, and the role of the material conditions of existence in a people's understanding of the world." (Duranti 1997: 4) The primary objectives in linguistic anthropological research revolve around the supposition that human language is a thinking faculty and, therefore, affects their actions/behaviour. The agency of language with an effect on human behaviour influences human habits, behaviour and culture. Therefore, it largely relies on extensive data of what human beings speak and write to study culture and behaviour (Duranti 1997). Such extensive characteristic of data to study the linguistic behaviour of people concerning an event is innately available in hashtags. Apart from the ability of hashtags in providing extensive data, the role of hashtags in social media is naturally relevant to the scope of linguistic anthropological studies. 
6 Further, hashtags carry emotive functions and perform as an expressing device. Saif M. Mohamed and Svetlana Kiritchenko (2015) have explored the word-emotion associations through hashtags and found that they can improve emotional classification accuracy in a different domain. Consisting of only a few words, the lexical structure of hashtags is unambiguous and straightforward. Various studies, including Giannoulakis \& Tsapatsoulis (2016), suggest that hashtags help accurately describe the intention of an image which can be inferred through image annotation processes like crowdsourcing.

7 The multidimensional features of hashtags hold the potentiality to participate as a reliable source scientifically and academically. Studies investigating the functions of hashtags find it helping in ideological construction, emotional connection and community building (La Rocca 2020; Colleoni 2013; Papacharissi 2015. Such functions of hashtags can further provide a linguistic window for anthropological studies, making hashtags perform as a reliable source for linguistic anthropology. Duranti (2003) has classified the evolution of linguistic anthropology into three paradigms. The first paradigm of linguistic anthropology is formalistic, focusing on the grammatical description, linguistic typology and classifications, where it shifts in the second paradigm to focus more on anthropology. The third paradigm is more anthropologically-oriented and brings up linguistic anthropological themes such as: investigations of personal and social identities, shared ideologies, construction of narrative interactions among individuals. Hashtags fall under the three themes above as they build social identity and a community of shared ideologies by creating narrative interactions among individuals.

\section{Collection of COVID-related Hashtags}

8 As set down by Hymes (1964: xxiii), the scope of linguistic anthropology can "include problems that fall outside the active concern of linguistics, and always it uniquely includes the problem of integration with the rest of anthropology." Duranti (1997: 4) has established that linguistic anthropology "does not mean that its research questions must always be shaped by the other subfields in anthropology." Therefore, the present study aims to study the potentiality and usability of hashtags in answering the anthropological questions related to COVID by studying the role of pandemic-related terms used in the hashtags, segmenting the hashtags lexically, studying the meaning and role of each word used in hashtags, and studying the socially and anthropologically concerned COVID-related neologisms found in hashtags.

Identifying the hashtags that are directly associated with the lifestyle of people during COVID-19 is a challenge. The virus has definitely brought unpredictable changes to our lives and also to the vocabulary that describes our lifestyles. The usage of words like quarantine, lockdown, pandemic have become more frequent in our daily lives along with corona and COVID after the outbreak (Katella 2020). Apart from them, the usage of phrases: oldnormal and newnormal to define the lifestyle pre- and post-COVID have become popular too. Several studies started to use the phrase 'new normal' for studying COVID and the lifestyle changes (Corpuz 2021; Cobianchi 2020; Xiao 2021). So, we decided to identify COVID-related hashtags using the presence of these seven words that were acknowledged to have been used in the context of pandemic in social media: Newnormal, Oldnormal, Corona, Quarantine, Lockdown, Pandemic. Also, the study labels 
these words as basetags, by which we mean the formation of hashtags using these words as a prefix.

We chose Instagram for our research as the medium to study the usage of COVIDrelated hashtags and its linguistic anthropological concerns. 87 hashtags were selected across the primary tags: \#newnormal, \#oldnormal, \#covid, \#corona, \#quarantine, \#lockdown, and \#pandemic. The hashtags across these primary tags were chosen based on their popularity (which is determined by the number of its occurrences) and the significance of the stemtags. The following are the hashtags chosen based on the data provided by Instagram in July 2021. The column with the number of occurrences notes its occurrences up until July 2021. A single post may include several occurrences of these hashtags. The number of occurrences of the hashtags here directly denote the popularity of hashtags and may not be directly proportional to the number of posts in Instagram. The chosen hashtags were collected based only on the selected primary tags and their popularity. The research does not attempt to bifurcate the hashtags spatially or temporally, a task rendered impossible by the data source. Therefore, the analysis of the listed hashtags focuses on the general impact of COVID-19 in social media through hashtags and its linguistic anthropological implications through Instagram.

Table 1: Chosen hashtags under the base tag \#newnormal

\begin{tabular}{|l|l|}
\hline Hashtag & Number of Occurrences \\
\hline \#newnormal & $5.4 \mathrm{M}$ \\
\hline \#newnormal2020 & $367 \mathrm{k}$ \\
\hline \#newnormallife & $98 \mathrm{k}$ \\
\hline \#newnormalwedding & $125 \mathrm{k}$ \\
\hline
\end{tabular}

Table 2: Chosen hashtags under the basetag \#oldnormal

\begin{tabular}{|l|l|}
\hline Hashtag & Number of Occurrences \\
\hline \#oldnormal & $5000+$ \\
\hline \#oldnormaldays & $500+$ \\
\hline \#oldnormalschool & $>100$ \\
\hline \#oldnormalvsnewnormal & $>100$ \\
\hline
\end{tabular}

Table 3: Chosen hashtags under the basetag \#quarantine

\begin{tabular}{|l|l|l|l|}
\hline Hashtags & Number of Occurrences & Hashtags & Number of Occurrences \\
\hline \#quarantine & $30.3 \mathrm{M}$ & \#quarantinefood & $312 \mathrm{k}$ \\
\hline
\end{tabular}




\begin{tabular}{|l|l|l|l|}
\hline \#quarantinelife & $12.7 \mathrm{M}$ & \#quarantineartclub & $304 \mathrm{k}$ \\
\hline \#quarantineandchill & $3 \mathrm{M}$ & \#quarantinekitchen & $224 \mathrm{k}$ \\
\hline \#quarantinecooking & $800 \mathrm{k}$ & \#quarantinestories & $182 \mathrm{k}$ \\
\hline \#quarantinebirthday & $653 \mathrm{k}$ & \#quarantinechallenge & $139 \mathrm{k}$ \\
\hline \#quarantineart & $629 \mathrm{k}$ & \#quarantinefitness & $127 \mathrm{k}$ \\
\hline \#quarantinedays & $546 \mathrm{k}$ & \#quarantinecuisine & $105 \mathrm{k}$ \\
\hline \#quarantinememes & $544 \mathrm{k}$ & \#quarantinemeals & $94.1 \mathrm{k}$ \\
\hline \#quarantineworkout & $451 \mathrm{k}$ & \#quarantineparty & $83.9 \mathrm{k}$ \\
\hline \#quarantinediaries & $340 \mathrm{k}$ & \#quarantinemoves & $63.3 \mathrm{k}$ \\
\hline \#quarantinebaking & $338 \mathrm{k}$ & \#quarantinegames & $59 \mathrm{k}$ \\
\hline \#quarantinemood & $317 \mathrm{k}$ & \#quarantineradio & $57.5 \mathrm{k}$ \\
\hline
\end{tabular}

Table 4: Chosen hashtags under the basetag \#lockdown

\begin{tabular}{|l|l|l|l|}
\hline Hashtags & $\begin{array}{l}\text { Number } \\
\text { Occurrences }\end{array}$ & of & $\begin{array}{l}\text { Number } \\
\text { Occurrences }\end{array}$ \\
\hline \#lockdown & $20.3 \mathrm{M}$ & \#lockdownactivities & $112 \mathrm{k}$ \\
\hline \#lockdown2020 & $4.7 \mathrm{M}$ & \#lockdownchallenge & $108 \mathrm{k}$ \\
\hline \#lockdownlife & $2.2 \mathrm{M}$ & \#lockdowndays & $107 \mathrm{k}$ \\
\hline \#lockdowndiaries & $406 \mathrm{k}$ & \#lockdownfitness & $82.9 \mathrm{k}$ \\
\hline \#lockdownart & $305 \mathrm{k}$ & \#lockdownrecipes & $77.1 \mathrm{k}$ \\
\hline \#lockdownmemes & $259 \mathrm{k}$ & \#lockdownsessions & $63.6 \mathrm{k}$ \\
\hline \#lockdowncooking & $238 \mathrm{k}$ & \#lockdownextended & $61.4 \mathrm{k}$ \\
\hline \#lockdownwedding & $196 \mathrm{k}$ & \#lockdowngardening & $41.1 \mathrm{k}$ \\
\hline \#lockdownbaking & $160 \mathrm{k}$ & \#lockdownhouseparty & $40.8 \mathrm{k}$ \\
\hline \#lockdownworkout & $130 \mathrm{k}$ & \#lockdownextension & $36.5 \mathrm{k}$ \\
\hline \#lockdownfood & $126 \mathrm{k}$ & \#lockdownmeals & $35 \mathrm{k}$ \\
\hline \#lockdownfun & $123 \mathrm{k}$ & \#lockdownagain & $20.8 \mathrm{k}$ \\
\hline \#lockdownphotography & $120 \mathrm{k}$ & & \\
\hline
\end{tabular}


Table 5: Chosen hashtags under the basetag \#covid

\begin{tabular}{|l|l|l|l|}
\hline Hashtags & Number of Occurrences & Hashtags & Number of Occurrences \\
\hline \#covid19 & $43.2 \mathrm{M}$ & \#covidkindness & $229 \mathrm{k}$ \\
\hline \#covid & $19.3 \mathrm{M}$ & \#covidcooking & $201 \mathrm{k}$ \\
\hline \#covidwedding & $477 \mathrm{k}$ & \#covid19news & $108 \mathrm{k}$ \\
\hline \#covidlife & $324 \mathrm{k}$ & \#covidiots & $80.3 \mathrm{k}$ \\
\hline \#covidart & $321 \mathrm{k}$ & \#covidphotodiaries & $70.5 \mathrm{k}$ \\
\hline \#covidmemes & $299 \mathrm{k}$ & \#covidclassics & $20.5 \mathrm{k}$ \\
\hline
\end{tabular}

Table 6: Chosen hashtags under the basetag \#pandemic

\begin{tabular}{|l|l|l|l|}
\hline Hashtags & Number of Occurrences & Hashtags & Number of Occurrences \\
\hline \#pandemic & $4.8 \mathrm{M}$ & \#pandemicwedding & $53.7 \mathrm{k}$ \\
\hline \#pandemic2020 & $674 \mathrm{k}$ & \#pandemicbirthday & $45 \mathrm{k}$ \\
\hline \#pandemiclife & $531 \mathrm{k}$ & \#pandemicbaking & $24.4 \mathrm{k}$ \\
\hline \#pandemicart & $114 \mathrm{k}$ & \#pandemiccooking & $22.1 \mathrm{k}$ \\
\hline \#pandemiccorona & $60.3 \mathrm{k}$ & \#pandemicoflove & $1000+$ \\
\hline \#pandemicparenting & $56 \mathrm{k}$ & & \\
\hline
\end{tabular}

Table 7: Chosen hashtags under the basetag \#corona

\begin{tabular}{|l|l|l|l|}
\hline Hashtags & Number of Occurrences & Hashtags & Number of Occurrences \\
\hline \#coronavirus & $35.8 \mathrm{M}$ & \#coronavirusmemes & $529 \mathrm{k}$ \\
\hline \#corona & $30.1 \mathrm{M}$ & \#coronawarriors & $208 \mathrm{k}$ \\
\hline \#coronamemes & $2.3 \mathrm{M}$ & \#coronavirusart & $46.5 \mathrm{k}$ \\
\hline \#coronatime & $1.4 \mathrm{M}$ & & \\
\hline
\end{tabular}

11 Further, to approach the COVID-related hashtags from an anthropological linguistic perspective, the study takes five of the ten variables that Herring (2007) suggested as the social facets of language on the internet. Herring (2007) suggested ten social facet variables: participation structure, participation characteristics, purpose, activities, 
topic, tone, norms of the organisation, norms of social appropriateness, norms of language, and code. The study designates five of the above facets:

- The purpose: to validate the significance of the words used in hashtags concerning the pandemic situations.

- The tone is the "manner or spirit of the conversation." Here, the study takes this variable to understand the nature of the neologisms and the emotive functions of the hashtags.

- Norms of the organisation: to categorise the hashtags based on their lexical features and the context.

- Participation characteristics: to discuss the sociolinguistic aspects. With a human as the participant, participation characteristics usually involves age, gender, cultural background, etc. Here, as the study uses hashtags as the participants, it studies the word order, genre and activities as the participation characteristics.

- Participation structure: the number of participants in an interaction. Our study employs this facet to evaluate the number of each hashtag on Instagram.

Duranti (1997) confirms that the role of linguistic anthropologists is scattered across the spaces of everyday encounters, language socialisation, ritual and political events, scientific discourse, verbal art, language contact, language shift, literacy events, and media. By applying Herring's facets to hashtags, the study verifies the scope of hashtags in the above mentioned areas of linguistic anthropology.

\section{Segmentation and Organization of Hashtags}

The lexical segmentation of hashtags aids in studying their linguistic and emotional characteristics. Different studies already suggest the methodology and framework for segmenting the hashtags for sociolinguistic (Qadir \& Elloff 2014) and computational reasons (Reuter et al. 2016; Berardi 2011). Qadir and Elloff (2014) have proposed a tree diagram method to break down the hashtags into individual lexical units for disambiguating and analysing the hashtags' emotional characteristics. Our current study segments the hashtags into stemtags and basetags through a similar tree diagram structure.

The study uses the term basetag to denote the pandemic-related terms found in hashtags and collects the hashtags associated with the basetag. The chosen basetags are \#newnormal, \#oldnormal, \#quarantine, \#lockdown, \#covid, \#pandemic, \#corona. Stemtags are the other significant words used in the hashtag, along with the basetag. Hence the hashtags are segmented into basetags and stemtags with a tree diagram structure, as shown in Fig. 1. The construction of the tree diagram is to help in categorising the stemtags into different genres and contexts. Also, it extends its role in understanding the relationship between the basetag and the stemtag. 
Figure 1. Segmentation of Simple Hashtags

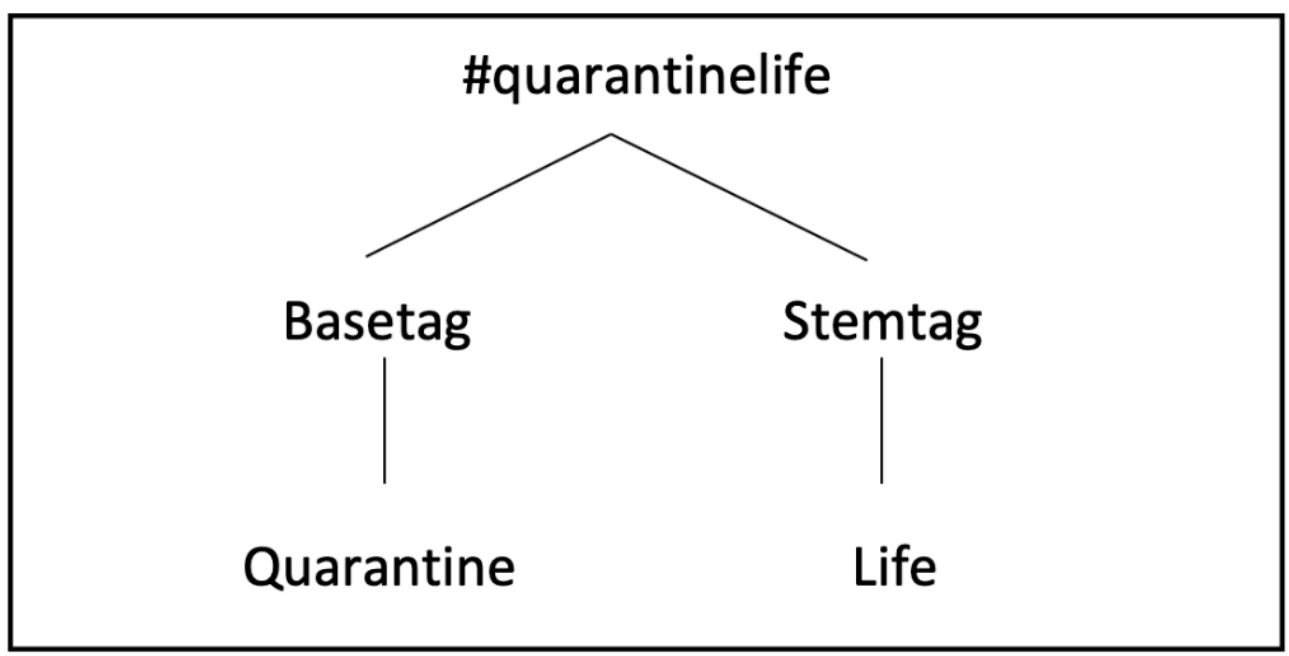

Source: The diagram was made by the authors to illustrate the segmentation for methodological purposes

15 While most of the hashtags can be classified into simple basetags and stemtags, a few hashtags are complex as they carry conjunction in them, as shown in Fig 2.

Figure 2. Segmentation of Complex Hashtags

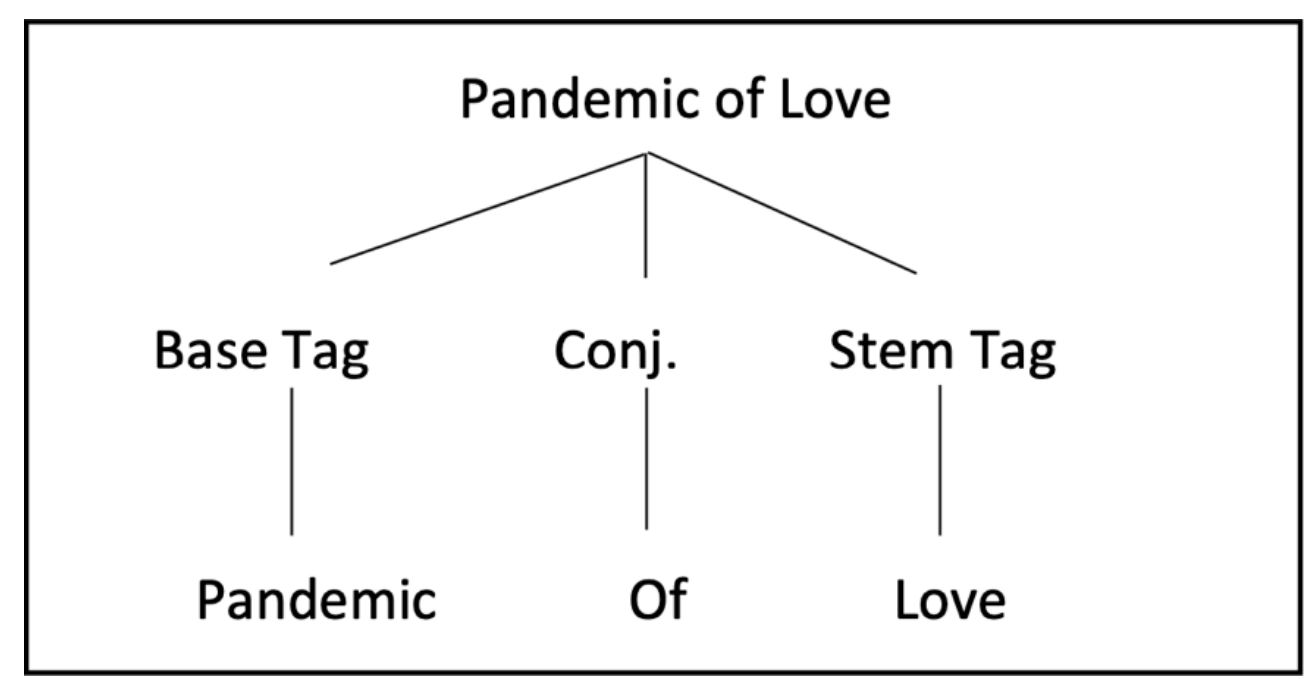

Source: The diagram was created by the authors to illustrate the segmentation for methodological purposes

The above example does not take the conjunction as a part of stemtag as we consider stemtag an exclusive content word intended to add a new aspect to the chosen basetags. In hashtags like \#coronavirus and \#pandemicorona, the basetag and stemtag are contextually synonymous. Therefore, they are treated as a single unit of a hashtag, excluded from being segmented into base and stem. The other hashtags are segmented into basetag and stemtag, and the stemtag of each hashtag is collected for further analysis, as shown in Image 1. 


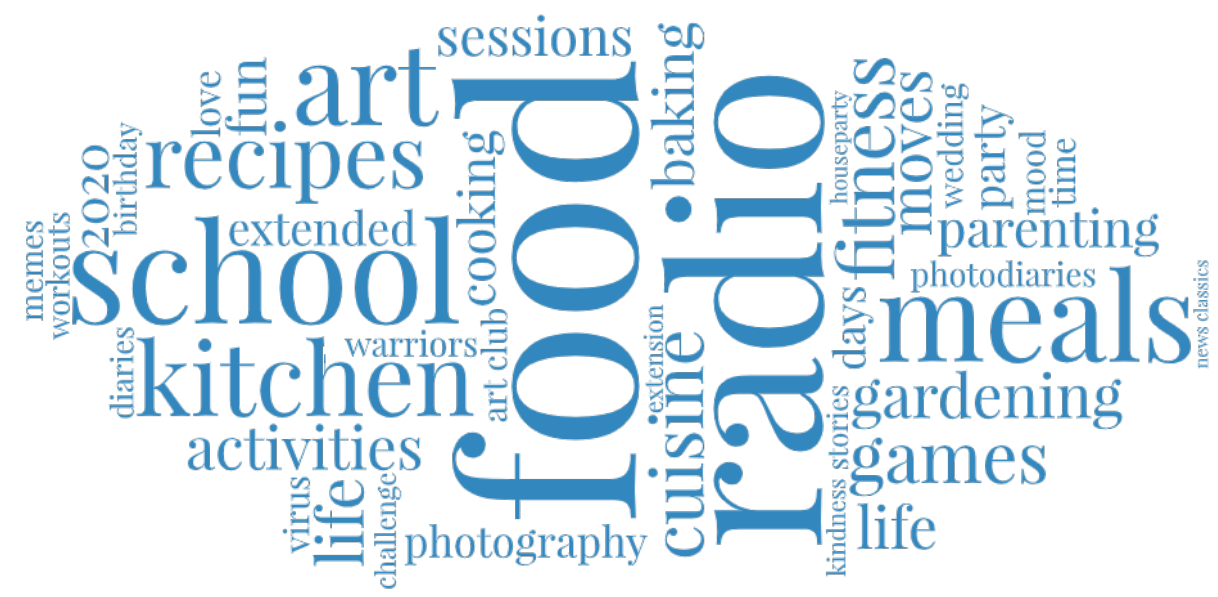

The wordcloud created post-segmentation by collecting all the stemtags related to different basetags. Source: The wordcloud was created after the segmentation of all hashtags with wordart.com

The stemtags are further categorized into distinctive stemtags and common stemtags. The specific stemtags under the distinctive category is exclusive and more accurate to the content. The distinctive stemtags are specific and unidimensional. These tags are directly associated with the content, while the common stemtags carry words that act as an umbrella term for a multitude of content. The distinctive stem tags include \#cuisine, \#fitness, \#gardening, whereas the common stemtags contain words like \#life, \#days and \#time that do not accurately describe the content of the post but are still relevant, as shown in images 2 and 3.

Image 2: Wordcloud of Common Stemtags

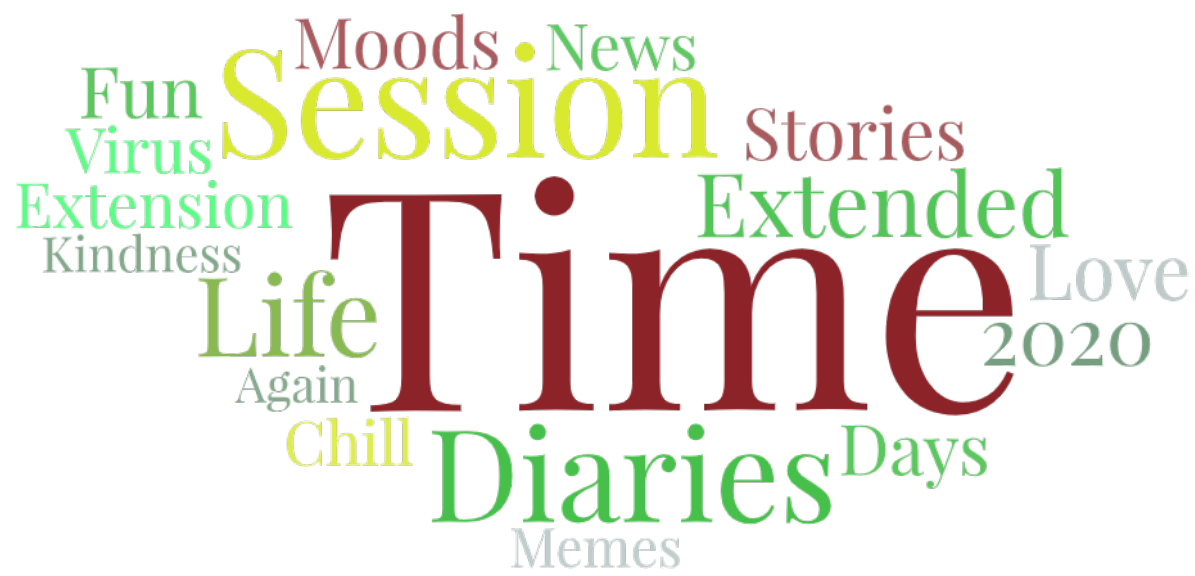

The wordcloud shows the further classification of stemtags called 'common stemtags' identified to represent generic content.

Source: The wordcloud was created after the segmentation and categorization with wordart.com 


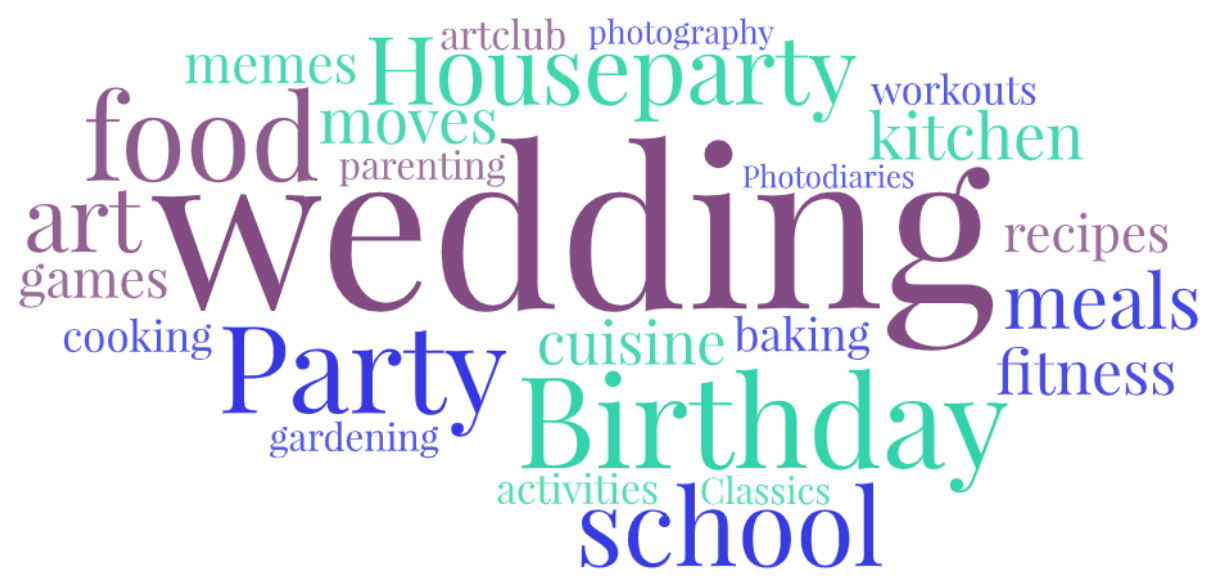

The wordcloud shows the further classification of stemtags called 'distinctive stemtags' identified to represent unidimensional or genre-specific content.

Source: The wordcloud was created through segmentation and categorization with wordart.com

\section{The COVID Lexicon}

From an anthropological perspective, the vocabulary of people reflects the lifestyle they have. J. K. Chambers argues that

People adjust their vocabulary, sounds, and syntax depending upon whom they are speaking to and the circumstances of the conversation. Such adjustments are often linguistically subtle and socially meticulous, and largely subconscious. They are not taught or consciously learned, but are part of the innate linguistic competence of all normal people. (Chambers 2007: 4601)

Chambers' argument in the sociolinguistic context is valid from an anthropological perspective too. The changes in people's vocabulary due to the circumstances is crucial for an anthropological understanding of the circumstances. This paper identifies the basetags as a representation of people's COVID lexicon. The study analyses the number of occurrences of basetag to identify the preferred quarantine-related word for hashtags in Instagram. 


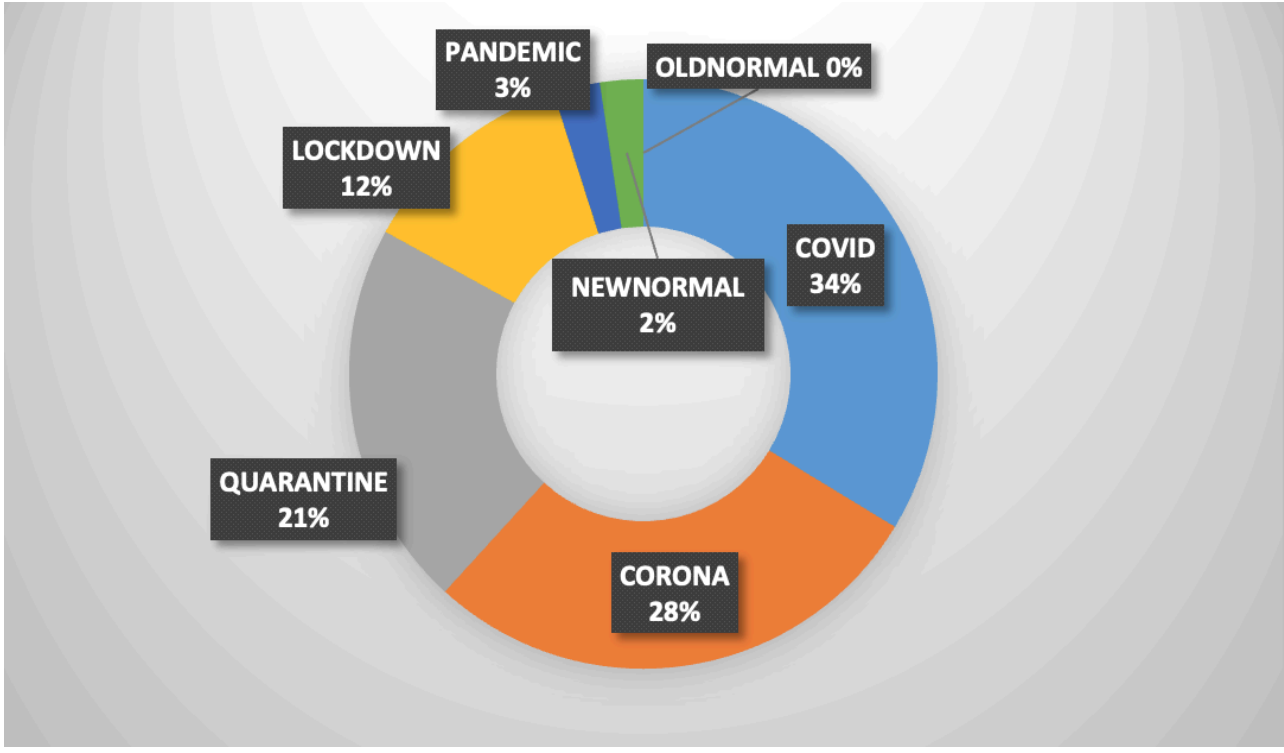

The pie chart depicts the distribution of occurrences of each basetags in the collected hashtags. The number of occurrences of each basetag from the collected data is calculated to study the most popular basetag during the pandemic. In the identified basetags, \#COVID was used in 34\% the Instagram hashtags and occurred a total of $83.8 \mathrm{M}$ times, \#corona was used in $28 \%$ of the hashtags and occurred $69.9 \mathrm{M}$ times, \#quarantine was in $21 \%$ of the collected hashtags with $52.8 \mathrm{M}$ total occurrences, \#lockdown was in $12 \%$ with a total of $30 \mathrm{M}$ occurrences, \#pandemic in $3 \%$ of the hashtags and occurred 6.38M times, \#newnormal in 2\% of the hashtags and occurred 5.9M times, and \#oldnormal in less than $1 \%$ of the hashtags and occurred only $5.7 \mathrm{k}$ times.

Source: The chart was created by the authors to show the distribution of the chosen basetags.

The two basetags \#newnormal and \#oldnormal may not be considered as words which can be directly related to the pandemic. But these basetags stand as a testimony to how the pandemic affected and re-defined normalcy. The adjective new in \#newnormal defines the sudden change in the way of living while the 'normal' in \#newnormal emphasizes why this sudden change in lifestyle has to be normalized due to circumstances. While \#newnormal represents the life during the pandemic, \#oldnormal is a reminder of the life that was once lived before the COVID-19 pandemic. On comparing the counts between \#newnormal (5.9M) and \#oldnormal (5.7k), we can find that Instagram recorded the life during the pandemic more frequently than throwing back to the nostalgic content of \#oldnormal living in the context of the pandemic.

\section{Hashtags and COVID-Lifestyle}

21 The unidimensional, distinctive stemtags are specific and directly associated with the content. The distinctive stemtags and associated hashtags are further analysed to verify the potentiality of hashtags through the collected sample in answering the COVID-related socio-anthropological questions such as:

1. How did the pandemic affect social events and gatherings?

2. What were the activities people were involved during the lockdown days?

3. Did people build a virtual community during the socially-distant lockdown days? 


\subsection{Hashtags related to Social Events}

In the collected hashtags, a few carry the \#newnormal definition of events that the pandemic has brought. The sudden change of existing ideas and norms of events due to the pandemic can be studied through this set of hashtags. Significantly, social events like weddings or parties have donned a new-normal definition due to the social distancing norms as weddings were restricted to 50 people or when New York Governor Andrew Cuomo made it legal to conduct internet weddings. The stemtags associated with social events like \#wedding, \#party, \#houseparty, and \#birthday carry a new normal definition with the presence of the COVID-related basetags.

Figure 3. Hashtags and social events

\section{\#lockdownwedding}

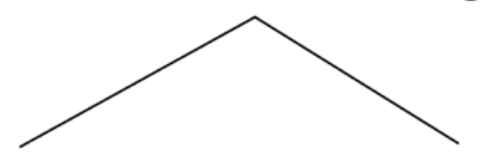

Basetag<smiles>C=CC=C</smiles>

Lockdown
Stemtag<smiles>CCCC</smiles>

Wedding

Source: The diagram was created by the researcher for segmentation purposes.

The segmentation conveys that hashtags like \#wedding, used for archiving or journaling a popular social event like a wedding, don a \#newnormal definition through the presence of the basetag. The use of the basetag \#lockdown before the stemtag \#wedding emphasizes the deviation of old norms in such social events and builds a new genre for the event \#wedding called a \#pandemicwedding, \#lockdownwedding, etc. The \#newnormal way of conducting social events is echoed in a few collected hashtags listed in Table 9.

Table 8: Examples of hashtags that carry a new pandemic-related identity to the existing events

\begin{tabular}{|l|l|}
\hline \#lockdownwedding & \#lockdownhouseparty \\
\hline \#pandemicwedding & \#quarantinebirthday \\
\hline \#pandemicbirthday & \#quarantineparty \\
\hline \#covidwedding & \#newnormalwedding \\
\hline
\end{tabular}




\subsection{Hashtags in Everyday Activities}

24 Other distinctive stemtags found mostly represent activities that can be directly associated with people's everyday lives. These set of hashtags can help understand the set of activities done by people as recorded in social media during COVID as shown in Table 10.

Table 9: Examples of hashtags that journals life during COVID

\begin{tabular}{|l|l|}
\hline \#pandemicart & \#pandemicbaking \\
\hline \#lockdownworkout & \#lockdowngardening \\
\hline \#lockdownmeals & \#covidphotodiaries \\
\hline \#lockdownrecipes & \#covidart \\
\hline \#pandemiccooking & \#pandemicparenting \\
\hline \#quarantinemeals & \#lockdownchallenge \\
\hline \#quarantineworkouts & \#lockdownactivities \\
\hline \#quarantinecooking & \#quarantinestories \\
\hline \#quarantinefood & \#quarantinegames \\
\hline \#quarantinebaking & \#quarantineradio \\
\hline \#quarantinekitchen & \#quarantineartclub \\
\hline \#quarantinecuisine & \#quarantinefitness \\
\hline
\end{tabular}

Further, the stemtags are categorized based on the activity they represent to find the most recorded activity by people on social media during COVID (Chart 2). 
Chart 2: Predominant activities recorded in social media based on the number of occurrences of the associated hashtags

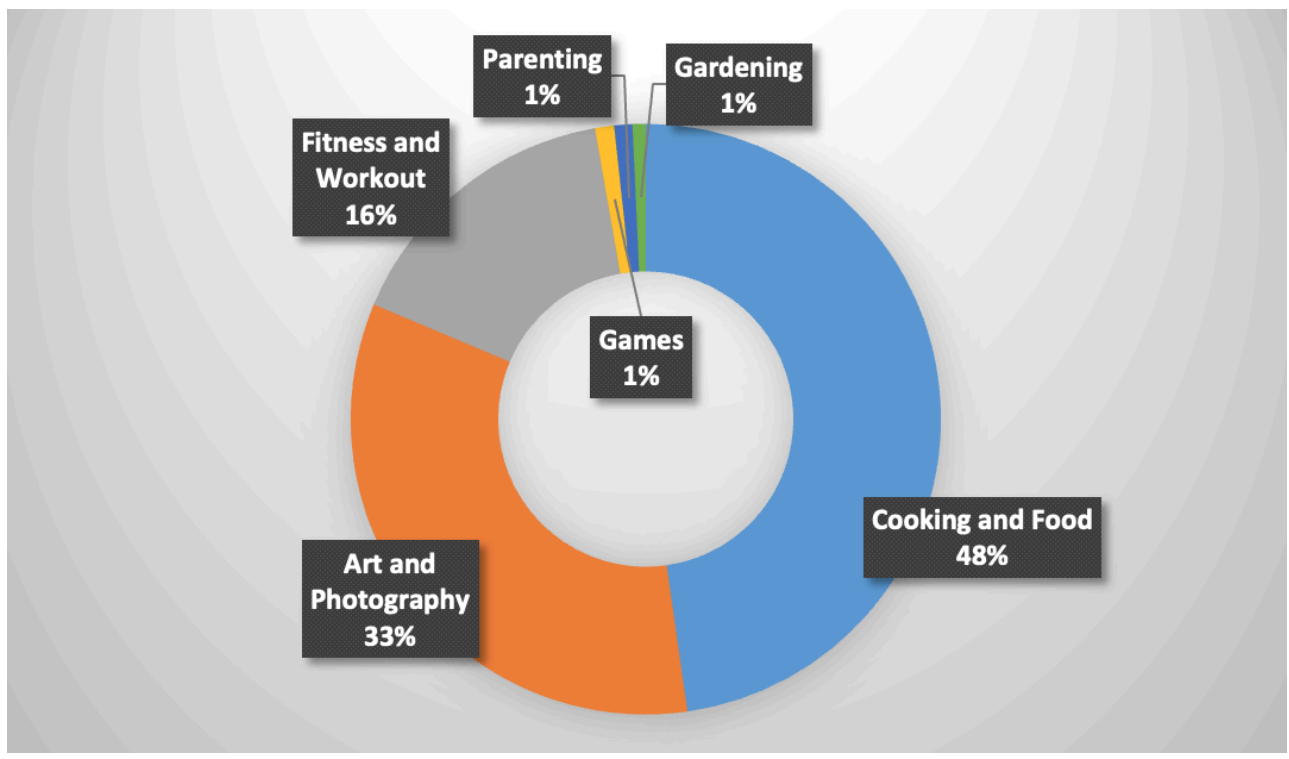

The chart explains that the majority of the activities-associated stemtags discuss cooking and food. $33 \%$ of the occurrences belong to art and photography related activities, and $16 \%$ fitness and workout. Stemtags related to gardening and games have contributed 1\% each in the occurrences of the collected activity-related stemtags.

Source: The chart was created by the authors to show the distribution of the stemtags related to daily activities recorded using hashtags in Instagram during COVID-19.

\subsubsection{Cooking and Food}

The posts on cooking and food on Instagram or any other social media has been popular even before the COVID period. The addition of the base tags help us identify the content in COVID times. The study focuses on food-related content during COVID by identifying the cooking and food-related stem tags. There is a multitude of stemtags related to cooking and food in the collected hashtags. They include \#cooking, \#recipes, \#meals, \#baking, \#kitchen, and \#food. From the collected data, the hashtags related to cooking and food has recorded over $2 \mathrm{M}$ occurrences, representing $48 \%$ of the total of hashtags referring to everyday activities.

Studying the hashtags related to everyday activities during COVID life with respect to their number of occurrences is a window to know the booming interests of people during lockdown. The analysis has provided us a clear picture about how food and art were prioritized during lockdown - work from home culture.

\subsubsection{Fitness and Workout}

The stemtags related to fitness and workout are \#workout and \#workouts. The hashtags related to fitness include \#lockdownworkout and \#quarantineworkouts. From the collected data, the hashtags related to fitness and workout has occurred over $899 \mathrm{k}$ times or $16 \%$ of the total. 


\subsubsection{Boardgames} lockdown period. The hashtag \#pandemicparenting occurred over 56k times on Instagram, or $1 \%$ of the total.

\subsection{Hashtags in Community Building}

Through using similar hashtags for a cause, hashtags indirectly create a community of social media users. It helps create affective publics, which is a term defined by Papacharissi (2015: 5) as "networked publics that are mobilized and connected, identified, and potentially disconnected through expressions of sentiment." Sometimes, hashtags build an idea of community by involving people to participate with a specified purpose through these hashtags actively. The stemtags \#artclub and \#challenges have been identified to perform the community-building function in the collected distinctive stemtags. These stemtags have collectively occurred over $551 \mathrm{k}$ times in the collected set of distinctive hashtags.

\section{Identified Functions of the Common Stemtags}

The study identifies common stemtags (see Image 3, above) that are generic and multidimensional. These are words that cannot be associated with a single activity or a specific content. Although the stemtags seem to be generic, further lexical analysis of the stemtags shows that they perform one of the three identified functions: archival, emotive and reactive (see Chart 3, below).

Further, the number of occurrences of each stemtag is compared and analysed under the hypothesis that the number of occurrences can help understand the most preferred performative hashtags' function during COVID. 


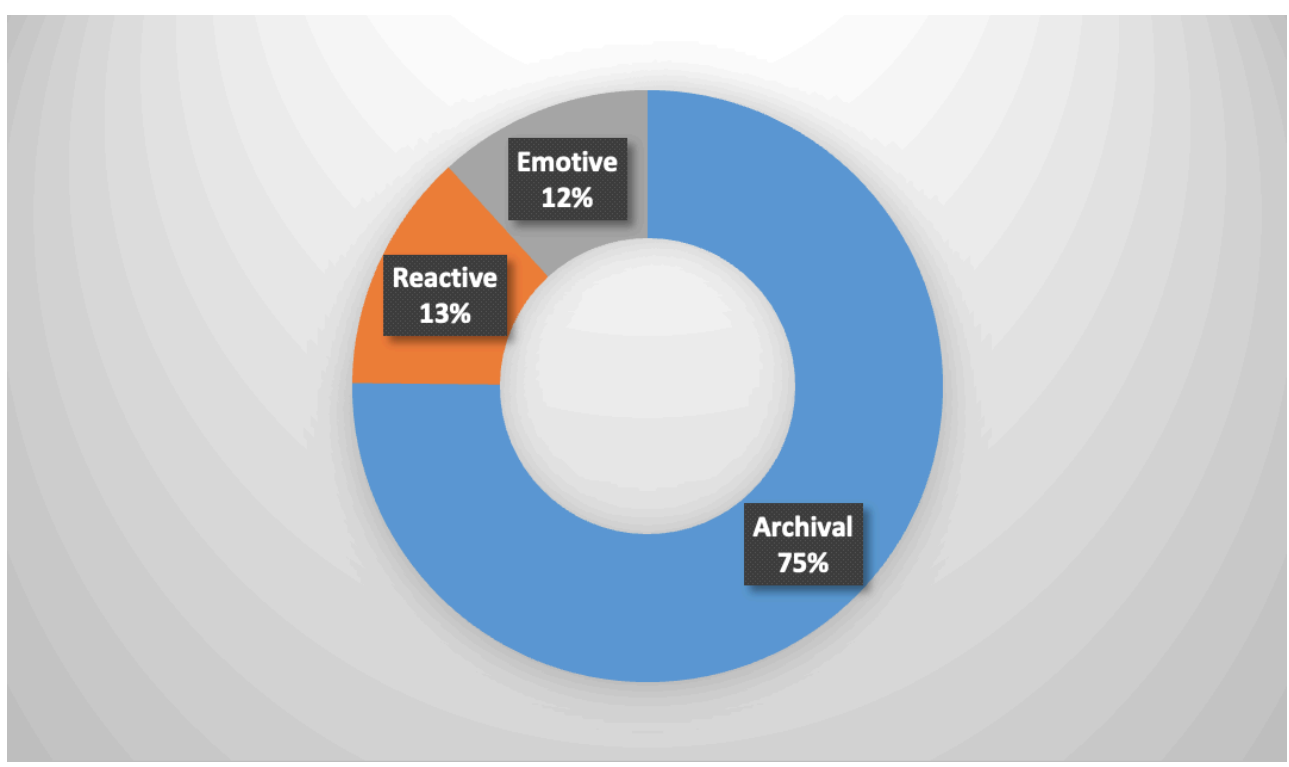

The analysis displays that $75 \%$ of common stemtags are used for archival or journaling functions, while $12 \%$ are intended to perform emotive functions and $13 \%$ for reactive functions. Also, this analysis acts as evidence of people's preference for using hashtags to 'record' their daily lives during COVID, which can be used as a source for anthropological studies.

Source: The chart was created by the authors to show the identified functions of stemtags and its distribution.

\subsection{Stemtags that perform archiving functions}

Stemtags like \#diaries, \#life, \#time carry a chronicling aspect to the quarantine-related hashtags. Through word choices like \#diaries or \#days, social media have consciously or unconsciously journaled life during COVID. The hashtags associated with such stemtags are called archival hashtags. Seven stemtags were identified to perform this function: \#2020, \#life, \#days, \#diaries, \#stories, \#sessions, and \#time.

\subsection{Stemtags that perform emotive functions}

Stemtags like \#fun, \#chill, \#kindness, \#love and \#mood can be associated with the emotive aspect of the content. Such stemtags act as a window to understanding the lockdown-mind space of people and bring affective/emotive characteristics to the hashtags.

\subsection{Stemtags that perform responsive functions}

Stemtags like \#extended, \#extension, \#again, and \#memes carry people's reaction to COVID-related lockdowns. Therefore, we identify them performing the reactive or responsive function as a hashtag to COVID and lockdown. 


\section{Neologisms in COVID}

COVID-19 has brought numerous new words to the dictionary, from words like Covidiots and Coronapocalypse that discuss COVID-related living, the \#newnormal has also brought strange neologisms including maskne, which is acne developed by the regular wearing of masks, and quaratini, which is a cocktail had in quarantine. AlAzzawi \& Haleem (2021) have listed neologisms related to the COVID-19 pandemic used in hashtags. Our study focuses on the number of occurrences of these neologisms in Instagram hashtags and further, study their popularity and functions. By number of occurrences, we mean the times of recurrences of the hashtags in Instagram and it may not be an equivalent to the number of posts. The following records a selection of new phrases and words formed during the pandemic.

Table 10: Examples of COVID-related neologisms in Instagram hashtags

\begin{tabular}{|c|c|c|c|}
\hline $\begin{array}{l}\text { S. } \\
\text { No. }\end{array}$ & $\begin{array}{l}\text { Neologisms in } \\
\text { Hashtags }\end{array}$ & Meaning & $\begin{array}{l}\text { Number of } \\
\text { Occurrences }\end{array}$ \\
\hline 1. & \#coronacation & $\begin{array}{l}\text { The break from regular \#oldnormal school and } \\
\text { workstyle that is treated as a vacation from regular } \\
\text { lifestyle. }\end{array}$ & $198 \mathrm{k}$ \\
\hline 2. & \#Maskne & Acne developed by wearing masks & $178 \mathrm{k}$ \\
\hline 3. & \#Quarantini & Any kind of a cocktail drunk in quarantine & $109 \mathrm{k}$ \\
\hline 4. & \#Coronapocalypse & $\begin{array}{l}\text { A word used to described the extreme social, } \\
\text { economic and political reactions to the pandemic. }\end{array}$ & $109 \mathrm{k}$ \\
\hline 5. & \#covidient & $\begin{array}{l}\text { Used for describing a person who follows the public } \\
\text { health guidelines to save themselves from covid. }\end{array}$ & $100 \mathrm{k}$ \\
\hline 6. & \#Pandemicbaby & A baby that was born during the pandemic & $97.1 \mathrm{k}$ \\
\hline 7. & \#Covidiot & $\begin{array}{l}\text { COVID+Idiot, used for describing people that do not } \\
\text { understand the seriousness of the pandemic or do } \\
\text { not follow the public health guidelines. }\end{array}$ & $53.9 \mathrm{k}$ \\
\hline 8. & \#quaranteen & $\begin{array}{l}\text { For recording the lifestyle of teen agers during the } \\
\text { quarantine. }\end{array}$ & $32.9 \mathrm{k}$ \\
\hline 9. & \#coronacoaster & Describing the ups and downs of pandemic. & $12.2 \mathrm{k}$ \\
\hline 10. & \#Infodemic & $\begin{array}{l}\text { Manipulation of information and inauthenticity } \\
\text { during the pandemic. }\end{array}$ & $1000+$ \\
\hline
\end{tabular}




\begin{tabular}{|l|l|l|l|}
\hline 11. & \#pandumbic & $\begin{array}{l}\text { For describing someone who is ignorant of the public } \\
\text { health guidelines or lacks knowledge about the } \\
\text { pandemic. }\end{array}$ & $500+$ \\
\hline
\end{tabular}

The study classifies these neologisms into contexts as describing the pandemic behaviour of people, describing lifestyle during the pandemic, describing the socio-political effects of the pandemic, and describing the pandemic in general. The hashtagged neologisms identified to describe people during the pandemic were \#pandumbic, \#covidient, \#quaranteen, \#pandemicbaby and \#covidiot. Neologisms related to the socio-political effects of the pandemic were \#infodemic and \#coronapocalypse. Neologisms discussing the lifestyle of people during the pandemic were \#quarantini, \#maskne, \#coronacation and \#coronacoaster.

The role of neologisms in hashtags can be further analyzed in two ways: 1. Based on the number of neologisms that occurred under each category. 2. Based on the frequency of occurrences of the neologisms under each category.

\section{Chart 4: Occurrences of Neologisms in hashtags based on their Categories}

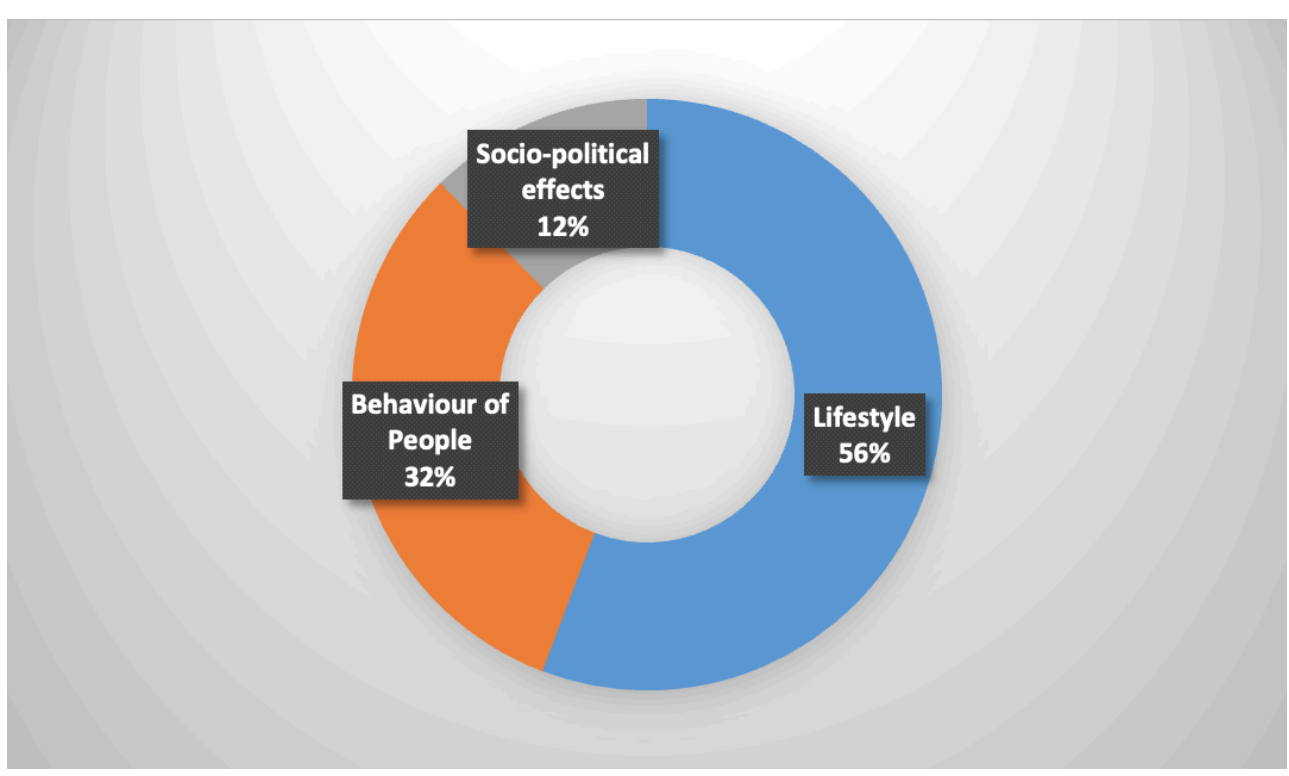

Based on the total number of occurrences, the lifestyle-related neologisms occupy $56 \%$ of the total occurrences, neologisms for describing people in the pandemic, $32 \%$, and neologisms related to sociopolitical effects, $12 \%$.

Source: The chart was created by the authors to show the distribution of functions of COVID-related neologisms.

\section{Conclusion}

Solving any anthropological conundrum accounts for its direct engagement with human behaviour and lifestyle. The validity of anthropological findings and speculations rely on the anthropologist's sources and primarily on the extension of first-hand anthropocentric information the anthropologist can collect. Therefore, it is significant for anthropological data to be vivid, immediate, and empirical to perform as a reliable research source.

42 By studying pandemic-related hashtags, we found that hashtags could record and describe people's lifestyles and social reactions vividly. This quality of hashtags make 
them an essential tool for digital anthropological studies. COVID-related hashtags brought a specific hashtag genre to the social media database, reflecting people's mind space during COVID. Other than just performing as an expressing device, hashtags can act as a digital repository for the times.

The linguistic analysis of the chosen COVID-related hashtags has provided a glimpse to the lifestyle of people during this period of crisis. The results show us that social media is frequently used during the pandemic for a multitude of purposes. The lexical combinations of the hashtags and their number of occurrences have allowed us to list the following findings with respect to the COVID lifestyle:

- The presence of the identified basetags as a prefix to several common and distinctive stemtags showed us the people's approach to the pandemic lifestyle as a specific genre.

- On comparing the counts between \#newnormal (5.9M) and \#oldnormal (5.7k), we found that Instagram recorded the life during the pandemic more frequently than throwing users back to nostalgic content of \#oldnormal living in the pandemic context.

- The study of the distribution of everyday activities in the hashtags showed that cooking and food had taken a predominant space during life in lockdown.

- The stemtags like \#challenges and \#club proved people's initiatives to digitally build communities in a phase in which social distancing became the norm.

Duranti affirms that

linguistic anthropologists start from the assumption that there are dimensions of speaking that can only be captured by studying what people actually do with language, by matching words, silences, and gestures with the context in which those signs are produced. (Duranti 1997: 9)

The current study focused on how people treated hashtags and their purpose. Through a linguistic analysis, we could find the following psycho-social dimensions of hashtags during the pandemic. The segmentation and the comparison of the total range of hashtags under each keyword helped us study people's lifestyle choices and preferences during COVID. Further, the frequency of the usage of pandemic-related words in hashtags helped us determine that these words have become more common in people's everyday vocabulary. Also, through the performative functions of stemtags, we found hashtags being preferred for people to journal or archive their own lives, which can make the study of hashtags provide first-person information in anthropological enquiries.

Segmenting hashtags and lexically analysing them provide significant help in visualising vivid imagery related to the situation. Also, the unambiguity and clarity in the hashtags make them a better repository of anthropological information, providing a psycho-social and anthropological perspective to people's lifestyles. Therefore, hashtags can be treated as a potential data source to study the anthropological aspects of a phenomenon.

Apart from a qualitative study of the hashtags through linguistic choices, the available quantitative details, based on the number of occurrences of a particular hashtag, can help understand lifestyle choices and other anthropological related information. For instance, studying the count of different hashtags that convey probably opposite meanings can help understand the stand of the majority in social media on a particular cause or event. The potentiality of hashtags in many linguistic pieces of research also extends their significance in studies across the arena of digital humanities. 


\section{BIBLIOGRAPHY}

Al-Azzawi, Quasim Obayes, and Hanin Ali Haleem. "Do you speak Corona?: Hashtags and Neologisms since the COVID 19 Pandemic Outbreak." International Journal of Linguistics, Literature and Translation 4(4) 2021: 113-22. DOI: 10.32996/ijllt.2021.4.4.12

Atkinson, Meera. "Hashtag \#Affect.” Capacious: Journal of Emerging Affect Inquiry, 1(2) 2018. DOI: 10.22387/CAP2018.15

Bashari, Benyamin, and Ehsan Fazl-Erzi. "Influential Post Identification on Instagram through Caption and Hashtag Analysis." Measurement and Control 53.3-4 (2020): 409-415. DOI: $10.1177 / 0020294019877489$

Bernard, Andreas. Theory of the Hashtag. Trans. Valentine A. Pakis. UK: Polity Press, 2019.

Berardi, Giacomo, Andrea Esuli, et.al. “ISTI @ TREC Microblog track 2011: Exploring the use of hashtag segmentation and text quality ranking”. In TREC. 2011. http://trec.nist.gov/pubs/trec20/ papers/NEMIS_ISTI_CNR.microblog.update.pdf

Cahapay, Michael B. “Trending phrases, names and hashtags related to COVID'19 crisis in the Philippines: The Language of Social Crisis." Muallim Journal of Social Science and Humanities 4(4) 2020: 1-9. DOI: $10.33306 / \mathrm{mjssh} / 91$

Cartier, Emmanuel. "Neoveille, Web Platform for finding and monitoring neologisms in monitor corpora." Neologica 13 (2019): 23-54.

Chambers, J.K. "Sociolinguistics." In The Blackwell Encyclopedia of Sociology. Ed. George Ritzer. Oxford: Blackwell, 2007. 4601-4.Chen, Emily, Kristina Lerman, and Emilio Ferrara. "Tracking social media discourse about the COVID-19 pandemic: Development of a public coronavirus twitter data set". JMIR public health and surveillance 6(2) 2020. DOI: 10.2196/19273

Cobianchi, Lorenzo, Luigi Pugliese, et.al. "To a new normal: Surgery and COVID-19 during the transition phase". Annals of Surgery 272 (2) 2020: e49-e51. DOI: 10.1097/SLA.0000000000004083

Colleoni, Elanor. "CSR communication strategies for organizational legitimacy in social media". Corporate communications: An international journal. 18(2) 2013: 228-48. DOI: $10.1108 / 13563281311319508$

Corpuz, Jeff Clyde G. "Adapting to the culture of 'new normal': An emerging response to COVID-19" Journal of Public Health. 43 (2) 2021: e344-5. DOI: 10.1093/pubmed/fdab057

Cui, Hao, and Janoz Kertesz. "Attention dynamics on the Chinese Social Media Sina Weibo during COVID-19 Pandemic." EPJ Data Science 10(8) 2021: 1-16. DOI: 10.1140/epjds/s13688-021-00263-0

Duranti, Alessandro. Linguistic anthropology. New York: Cambridge UP, 1997.

Duranti, Alessandro. "Language as Culture in U.S. Anthropology: Three SParadigms." Current Anthropology 44.3 (2003): 323-347. DOI: 10.1086/368118

Duranti, Alessandro, ed. Companion to Linguistic Anthropology. Malden, MA: Blackwell, 2004.

Giannoulakis, Stamatios, and Nicolas Tsapatsoulis. "Evaluating the descriptive power of Instagram hashtags". Journal of innovation in Digital Ecosystems. 3(2) 2016: 114-29. DOI: 10.1016/ j.jides.2016.10.001

Goold, Kari L. J., Reynafe N. Aniga, Peter B. Gray. "Sports Under Quarantine: A Case Study of Major League Baseball in 2020.” Social Sciences 10(1): 5. 2020:1-14. DOI: 10.3390/socsci10010005 
Han, Yoojin, and Hyunsoon Lee. "Lifestyle experiences: Exploring key attributes of lifestyle hotels using Instagram user-created content in South Korea." Sustainability 13(5). 2021: 1-18. DOI: $10.3390 /$ su13052591

Herring, Susan C. “A faceted classification scheme for computer mediated discourse.” Language @ Internet, 4. 2007. https://www.languageatinternet.org/articles/2007/761

Highfield, Tim, and Tama Leaver. “A methodology for mapping Instagram hashtags.” First Monday, 1-5(20). 2015. DOI: 10.5210/fm.v20i1.5563

Hussain, Wajahat. "Role of Social Media in COVID-19 Pandemic". International Journal of Front Sciences 4(2) 2020: 59-60. DOI: 10.37978/tijfs.v4i2.144

Hymes, Dell H. “The anthropology of communication." In Human communication theory: Original essays. Ed. F.E. Dance. New York: Holt, Rinehart and Winston, 1967. 1-33.

Hymes, Dell H. "Models of the interaction of language and social setting." Journal of Social Issues, 23(2) 1967: 8-28.

Hymes, Dell H., ed. Language in Culture and Society: A Reader in Linguistics and Anthropology. New York: Harper \& Row, 1964.

Hymes, Dell H. Essays in the History of Linguistic Anthropology. Amsterdam/Philadelphia: John Benjamins Publishing Company, 1983.

Ibba, Simon, Matte Orru, et al. "Hashtag of Instagram: From Folksonomy to Complex Network." In Proceedings of the 7th International Joint Conference on Knowledge Discovery, Knowledge Engineering and Knowledge Management (IC3K 2015) (2) 2015: 279-284.

Katella, Kathy. "Our new COVID-19 vocabulary - What does it all mean?" Yale Medicine. 2020. http://www.yalemedicine.org/news/covid-19glossary

Krek, M. Langements, etal. "Electronic Lexicography in the $21^{\text {st }}$ Century: Thinking outside the paper." Proceedings of eLex. Ljubljana. Tallinn: Trojina, Institute for Applied Slovene Studies. 2011.

La Rocca, Gevisa. "Possible selves of a hashtag: Moving from the theory of speech acts to cultural objects to interpret hashtags". International Journal of Sociology and Anthropology. 12 (1) 2020: 1-9. DOI: $10.5897 /$ IJSA2019.0833

Latour, Bruno. Reassembling the Social: An Introduction to Actor Network Theory. Oxford: Oxford UP, 2005.

Lee, Carmen, and Dennis Chau. "Language as pride, love and hate: Archiving Emotions through multilingual Instagram hashtags.” Discourse, Context and Media 22 (2017): 21-29. DOI: 10.1016/ j.dcm.2017.06.002

Mohammad, Saif, and Svetlana Kiritchenko. "Using hashtags to capture fine emotion categories from tweets.” Computational Intelligence 31(2) 2015: 301-326. DOI: 10.1111/coin.12024

Nikjoo, Adel, Bardia Shabani, and Ana Beatriz Hernández-Lara. "What do people share from quarantine?" Current Issues in Tourism 24(14) 2020: 1965-1969. DOI: 10.1080/13683500.2020.1808598

Papacharissi, Zizi. "Affective publics and structures of storytelling: sentiment, events and mediality." Information, Communication \& Society 19.3 (2015): 307-324. DOI: 10.1080/1369118X. 2015.1109697

Priadana, Adri, Sylvert Prian Tahalea. "Hashtags Activism and Message Frames: Social Network Analysis of Instagram during COVID-19 Pandemic Outbreak in Indonesia." Journal of Physics: Conference Series. 2021. DOI: 10.1088/1742-6596/1836/1/012031 
Qadir, Ashequl, and Ellen Riloff. "Learning Emotional Indicators from Tweets: Hashtags, Hashtag patterns and Phrases." In Proceedings of the 2014 Conference on Empirical Methods in Natural Language Processing (EMNLP). 2014: 1203-1209. DOI: 10.3115/v1/D14-1127

Reuter, Jack, Jhonata Pereira-Martins, and Jugal Kalita. "Segmenting Twitter Hashtags.” International Journal on Natural Language Computing 5(4) 2016: 23-36. DOI: 10.5121/ijnlc.2016.5402

Scott, Kate. "The Pragmatics of Hashtag: Inference and Conversation Style on Twitter." Journal of Pragmatics 81 (2015): 8-20. DOI: 10.1016/j.pragma.2015.03.015

Shanthakumar, Swaroop Gowdra, Anand Seetharaman and Arti Ramesh. "Understanding the socio-economic disruption in the United States during COVID-19 early days". Preprint. 2020. https://arxiv.org/abs/2004.05451

Tan, Kim Hua, Peter Woods et.al. "COVID-19 Insights and Linguistic Methods”. 3L: The Southeast Asian Journal of English Languge Studies. 26(2) 2020: 1-23. DOI: 10.17576/3L-2020-2602-01

Tasnim, Samia, Md Mahbub Hossain, and Hoimonty Mazumder. "Impact of Rumour and Misinformation on COVID-19 in Social Media." Journal of Preventive Medicine and Public Health. 53(3) 2021: 171-4. DOI: 10.3961/jpmph.20.094

Wood, Johnny. "This is how couples around the world are marrying under COVID-19" World Economic Forum. 2020. http://www.weforum.org/agenda/2020/04/coronavirus-covid-19wedding-online/

Whiting, Anita, and David Williams. "Why people use social media: a uses and gratifications approach." Qualitative market research: An International Journal 16(4) 2013: 362-9. DOI: 10.1108/ QMR-06-2013-0041

Xiao, Junhong. "Decoding new normal in education for the post COVID-19 world: Beyond the digital solution." Asian Journal of Distance Education. 16(1) 2021: 141-55. DOI: 10.5281/zenodo. 4818369

Xiong, Ying, Moonhee Cho, and Brandon Boatwright. "Hashtag activism and message frames among social movement organization: Semantic Network analysis and thematic analysis of twitter during the \#metoo movement." Public Relations Review. 45(1) 2018: 10-23. DOI: 10.1016/ j.pubrev.2018.10.014

Xi, Wanyu, Wenqian Xu, Xin Zhang, Liat Ayalon. "A Thematic Analysis of Weibo Topics (Chinese Twitter Hashtags) Regarding Older Adults during COVID-19 Outbreak." Journals of Gerontology: Series B 76(7)2020: e306-e312. DOI: 10.1093/geronb/gbaa148

Zyot, Fadi Ali Abdel-Qadar, Abeer Al-Gazo. "The most common used hashtags on facebook during the corona pandemic among Jordanians: A case study". International Journal of Language and Linguistics 8(1) 2021: 43-56. https://ijllnet.com/journal/index/2635 DOI: 10.30845/ijll.v8n1p6 Zappavigna, Michele. "Searchable talk: the linguistic functions of hashtags." Social Semiotics 25(3). 2015: 274-291. DOI: 10.1080/10350330.2014.996948

\section{ABSTRACTS}

Hashtags in social media reflect the Coronavirus pandemic outbreak and the consequential shifts and swifts in people's lifestyles. Several studies related to the pandemic have used hashtags from linguistic, economic, and sociological perspectives. However, the potentiality of hashtags in addressing the pandemic-related anthropological questions is still underexplored. This study verifies how hashtags are primarily anthropocentric and can act as a source for anthropological 
studies. We have collected COVID-related hashtags in Instagram under five different pandemicrelated words like \#newnormal, \#oldnormal, \#quarantine, \#lockdown, \#pandemic, \#corona. Eighty-eight hashtags under the mentioned pandemic-related keywords and 11 neologisms are collected to analyse their linguistic patterns and anthropological implications. The hashtags are segmented into basetags and stemtags to find the lexical significance of individual word units in the pandemic lifestyle. The segmentation helped analyse the range of vocabulary in hashtags used to describe people's lifestyles during the pandemic. Hashtags are user-friendly and are primarily used as an expressing and recording device. The archival and emotive quality added to hashtags' searchability and accessibility make them a potential linguistic anthropological research source for the 'COVID-19 pandemic.

Les hashtags dans les médias sociaux reflètent l'épidémie de pandémie de coronavirus et les changements qui en découlent dans le mode de vie de la population. Plusieurs études liées à la pandémie ont utilisé les hashtags dans une perspective linguistique, économique et sociologique. Cependant, le potentiel des hashtags pour aborder les questions anthropologiques liées à la pandémie est encore peu exploré. Cette étude démontre que les hashtags sont avant tout anthropocentriques et peuvent servir de source pour les études anthropologiques. Nous avons collecté les hashtags liés au COVID sur Instagram sous cinq mots différents liés à la pandémie comme \#newnnormal, \#oldnormal, \#quarantine, \#lockdown, \#pandemic, \#corona. Quatre-vingthuit hashtags liés aux mots-clés associés à la pandémie sus-mentionnés et 11 néologismes ont été collectés pour analyser leurs modèles linguistiques et leurs implications anthropologiques. Les hashtags sont segmentés en basetags et stemtags afin de trouver la signification lexicale des unités de mots individuels dans le mode de vie en période de pandémie. La segmentation a permis d'analyser l'étendue du vocabulaire des hashtags utilisés pour décrire le mode de vie des gens pendant la pandémie. Les hashtags sont conviviaux et sont principalement utilisés comme moyen d'expression et comme dispositif de consignation ou d'archivage. La qualité archivistique et émotive, ajoutée à la facilité de recherche et à l'accessibilité des hashtags, en font une source potentielle de recherche anthropologique linguistique pour analyser la pandémie de COVID-19.

\section{INDEX}

Mots-clés: hashtag, Instagram, COVID-19, linguistique anthropologique, Internet

Keywords: hashtag, Instagram, COVID-19, linguistic anthropology, Internet

\section{AUTHORS}

\section{SHRI VAISHALI}

Research scholar from VIT, Vellore, working on a comparative study between Internet Linguistics and the orthographic rules of Tholkappiyam. Department of English, School of Social Sciences and Languages, VIT, Vellore, India. Orcid id: 0000-0001-7843-9521. Contact: venkatshrivaishali [at] gmail.com

\section{S. RUKMINI}

Dr. S. Rukmini is Sr. Assistant Professor of English at VIT, Vellore. She has presented and published over 30 research articles and projects. Her specialization is related to a cross-cultural approach towards Indology and Digital Humanities. Department of English, School of Social Sciences and Languages, VIT, Vellore, India. Orcid Id: 0000-0001-8414-3145. Contact: rukminikrishna123 [at] gmail.com 